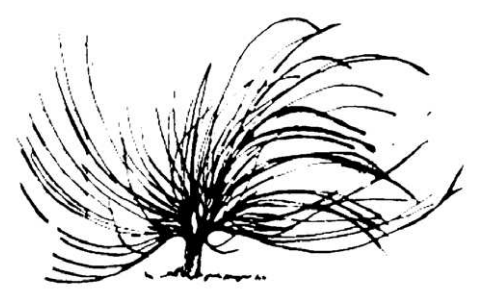

\title{
La otra cara de los programas de estudio: un análisis epistemológico del programa ética, estética $y$ ciudadanía de educación cívica
}

\author{
Mainor Andrés Campos Arce ${ }^{l}$ \\ Proyecto de Educación Abierta \\ Centro Educativo Quebradas \\ Alajuela, Costa Rica \\ ain.144@hotmail.com
}

\begin{abstract}
Resumen
La producción del conocimiento depende de los factores sociales, económicos, políticos, culturales, individuales e incluso colectivos y el uso de una teoría filosófica en particular sobre la producción del conocimiento, será la que determine qué tipo de conocimiento y qué resultados se quiere obtener de su aplicación. En Costa Rica, el Ministerio de Educación Publica (MEP) dentro de sus funciones debe promulgar los programas de estudio que cada disciplina utilizará e implementará, para ello busca sustento en teorías filosóficas que den respuesta a la manera de enseñar, aprender y crear conocimiento a través de la escuela. No obstante, existen principios dentro de algunas de estas teorías que esconden algunos postulados sombríos.
\end{abstract}

Palabras clave: epistemología, programas de estudio, globalización, estudiante, docente

Recibido: 1 de abril de 2013 - Aprobado: 11 de octubre de 2013

1 Bachiller en Enseñanza de los Estudios Sociales y Educación Cívica de la Universidad Nacional. Estudiante de la Licenciatura en Pedagogía con Énfasis en Didáctica de la Universidad Nacional. Actualmente se encuentra trabajando en Proyecto de Educación Abierta Centro Educativo Quebradas. 


\begin{abstract}
The production of knowledge depends on social, economic, political, cultural, individual, and even collective factors, and the use of a philosophical theory about the production of knowledge in particular will determine which type of knowledge and which results are to be obtained with its application. In Costa Rica, the Ministry of Public Education (MEP, for its acronym in Spanish), among its functions, must establish the syllabi that each discipline will use and implement. To do this, it must seek support in philosophical theories that respond to the way of teaching, learning, and creating knowledge through school. Nonetheless, there are principles in some of these theories that hide some dark propositions.
\end{abstract}

Keywords: epistemology, syllabi, globalization, student, teacher

E n este ensayo, se analizarán las diferentes teorías epistemológicas que se utilizan dentro del Programa Ética, Estética y Ciudadanía, para demostrar que estos planes no son consecuentes con lo establecido dentro de sus fines y que no cumplen con veracidad lo que dentro de su discurso se propone, de tal manera que se disfraza el objetivo verdadero de lo que se desea lograr mediante la puesta en práctica de estos planes de estudio.

La Epistemología es una rama de la Filosofía encargada de estudiar el conocimiento, de dónde surge, con qué fines y cuáles son su resultados, "la epistemología, o filosofía de la ciencia, es la rama de la filosofía que estudia la investigación científica y su producto, el conocimiento científico" (Bunge, 1981, p.1) en ese sentido, esta área filosófica se vuelve fundamental para determinar que tipo de conocimiento debe estar presente dentro de todas aquellas practicas educativas.

La producción del conocimiento depende de los factores sociales, económicos, políticos, culturales, individuales e incluso colectivos de los individuos, según la corriente epistemológica que se utilice, ya que esta será la que determine qué tipo de conocimiento y qué resultados se quiere obtener de su aplicación. En Costa Rica, el Ministerio de Educación Pública (MEP) es la institución encargada de garantizar, supervisar y dirigir 
el sistema educativo, dentro de sus funciones está el promulgar los programas de estudio que cada disciplina utilizará e implementará.

Para la creación de los programas de estudio, el MEP busca sustento en teorías filosóficas que den respuesta a la manera de enseñar, aprender y crear conocimiento a través de la escuela y la implementación de las políticas educativas inmersas en sus programas de estudio. No obstante, existen principios dentro de algunas de estas teorías que esconden algunos postulados sombríos.

Ante este panorama, el objetivo de este ensayo es analizar las diferentes teorías epistemológicas que se utilizan dentro del Programa Ética, Estética y Ciudadanía que se utiliza en la asignatura de educación ciudadana para los estudiantes de secundaria en Costa Rica. La razón fundamental es descubrir si estos planes son o no consecuentes con lo establecido dentro de sus fines y si cumplen verdaderamente con lo que dentro de su discurso se propone.

En el artículo 2 de la Ley Fundamental de Educación Costarricense, se estipulan una serie de fines, en otras palabras, intenta definir mediante una serie de enunciados, el resultado que pretende obtener con aplicación de las políticas educativas que se encuentran tanto en la ley como en los programas de cada una de las disciplinas que se brindan en primaria y secundaria. Algunos de estos fines son los siguientes:

- La formación de ciudadanos amantes de su patria, conscientes de sus derechos y de sus libertades fundamentales, con profundo sentido de responsabilidad y de respeto a la dignidad humana,

- Contribuir al desenvolvimiento pleno de la personalidad humana,

- Formar ciudadanos para una democracia en que se concilien los intereses del individuo con los de la comunidad,

- Estimular el desarrollo de la solidaridad y de la comprensión humana.

Mediante la incorporación de toda una gama de contenidos que encaucen hacia los cumplimientos de estos fines, el programa Ética, Estética y Ciudadanía menciona claramente sus pretensiones sobre cómo o que quieren crear cuando se aplique este programa a los y las estudiantes de secundaria, es decir, el perfil de salida que tendrán los graduados bajo este programa, "queremos que los estudiantes aprendan lo que es relevante y que lo aprendan bien: que nuestros jóvenes adquieran y desarrollen el conocimiento, la sensibilidad y las 
competencias científicas; lógicas y matemáticas; históricas y sociales"(M.E.P., 2009, p.5).

Lo anterior claramente refleja que dentro de este programa hay una marcada influencia del idealismo, principalmente el esencialismo pedagógico, ya que esta teoría, defiende los contenidos como "puros", o sea, como aprendizajes invariables e indefectibles; además, se puede mencionar que estos contenidos son aquellos que en su mayoría las clases altas dominantes han fijado como lo únicos que se puede considerar científicos y capaces de generar cierta relevancia. Pero, no solo se puede hablar sobre el estudiante, ya que bajo este paradigma epistemológico el docente tiene también un papel que ocupar, y es cumplir la tarea de reproducir estos contenidos de la forma más fiel posible, de modo que el estudiante tendría un papel pasivo aprendiendo como verdades incuestionables lo enseñado. Se da énfasis en potenciar el contenido, el estudiante no es lo relevante ni el verdadero motivo de la enseñanza, no se contempla el conocimiento previo que los alumnos puedan poseer, y mucho menos se pretende que el estudiante llegue a desarrollar una capacidad reflexiva o crítica ante los contenidos que se le están -prácticamente- imponiendo.

Se evidencia cómo el estudiante se constituye como objeto inactivo, y el docente, el individuo activo o el único poseedor del conocimiento; por lo cual fundamentalmente, la acción de aprender y enseñar dentro de esto será netamente individualista y la superación escolar radicará en quien pueda reproducir lo aprendido de una manera invariable a la forma en que se le enseñó.

Con lo expuesto anteriormente, se puede asociar otra corriente epistemológica, el dogmatismo, el cual postula que lo que se percibe y observa es lo real, es decir, lo enseñado en la escuela es legítimo e indudable, y los estudiantes no podrán entrar en contradicción ni mucho menos emitir alguna reflexión sobre lo que el docente les esta "enseñando" porque los contenidos son estáticos y estandarizados para cualquier parte del país, sin tomar en cuenta la cultura, el entorno o la cosmovisión de la sociedad en que se está implementando el programa, lo que convierte el acto de enseñar en una simple relación estudiante- profesor, lo que conlleva consecuencias adversas hacia la materia y hacia el deseo de permanecer en el aula por parte del estudiante, ya que al ser aplicados por igual para todos y todas, no se toman en cuenta los intereses $\mathrm{y}$ las necesidades de los cada uno de ellos. 
El implementar los mismos planes de estudio, sin tomar en cuenta las diferencias culturales, étnicas, religiosas, entre otras, es una manera de homogeneizar y también de excluir a aquellos que no se enmarcan dentro del modelo de estudiante que pretende el sistema educativo, "los niños que 'fracasan' en la escuela, no se debe necesariamente a un 'déficit' intelectual sino más bien a la interacción entre esas condiciones señaladas, mismas que se pueden superar o mejorar socialmente a través de la intervención política y ética, evitando con ello la 'construcción del fracaso escolar', que no es otra cosa que la exclusión social"(Gómez, 2012 , s.p.).

Según los encargados de la elaboración del programa, para la creación de este, "se toma en cuenta mediante la planificación y ejecución del currículo el conocimiento de la realidad y la problemática de la comunidad y la interacción permanente que existe entre el individuo y su entorno sociocultural" (M.E.P, 2009, p.18), lo cual hace preguntarse, si verdaderamente se toma en cuenta los contextos socioculturales, por qué no hay programas específicos para aplicar en los pueblos indígenas de nuestro país y únicamente se toman en cuenta estos grupos étnicos en un solo contenido de octavo año, dicho sea de paso lo que desea lograr es "interés por promover las manifestaciones artísticas y estéticas de la interculturalidad" (MEP 2009, p.98). Está por demás mencionar que no se contempla la manera en que estos grupos generan su conocimiento, ni su idioma, ni su historia, lo cual, como se notará más adelante, dejará a este programa fuera de la teoría socio-constructivista, en la cual dice estar enmarcado.

Esto lleva a analizar la metodología que tradicionalmente propicia el MEP,la cual no se interesa en que los estudiantes logren analizar, abstraer y sintetizar la información, por el contrario, la memoria es su base, "el dogmatismo no respeta al individuo y tampoco a la sociedad, la verdad o información es tal cual se muestra y se debe de aceptar y concebir de manera incuestionable" (Gómez, 2012, s.p.), lo que posiciona al docente como centro de todo el proceso de enseñanza, por ende, promoviendo el adulto centrismo.

Otra corriente epistemológica que se encuentra dentro del Programa Ética, Estética y Ciudadanía es la del Pragmatismo, la cual sostiene concretamente que "lo que tiene valor sobre todo es la utilidad y el valor práctico de las cosas" (Gómez, 2012, s.p.). Asimismo, esta corriente mantiene que la verdad y la bondad deben ser medidas de acuerdo con 
el éxito que se tenga en la práctica, por lo que, con la implementación de los proyectos de cívica, se logrará demostrar si verdaderamente los estudiantes aprendieron los contenidos mediante su puesta en práctica con éxito, "para eso debemos educar: tanto para la convivencia eficiente, útil y práctica del mundo del trabajo, del comercio o del consumo, como para la vida plena y trascendente que surge de la convivencia solidaria, del afecto desinteresado y de la responsabilidad con el medio" (M.E.P, 2009, p.4), en otras palabras, si algo no tiene un fin o uso determinado no hay razón para que tal cosa exista, lo que demuestra que estos programas solo pretenden educar para una convivencia útil, práctica y eficiente de un mercado laboral y no para brindar habilidades para la vida a los y las estudiantes de secundaria en el país.

Por otra parte, es importante notar que existe una contradicción evidente, pues dentro del mundo de la globalización, la lógica del mercado y el consumo, no promueven valores como la solidaridad, y mucho menos, una convivencia responsable para con el medio, "los jóvenes son una especie de no sujeto o una persona sin identidad y sin historia, son seres en potencia, en evolución, todavía no son, son una copia de su esencia (el adulto), llegarán a ser sólo si se dejan moldear, si adquieren las habilidades y destrezas necesarias para ser en el futuro (ser en el mercado)" (Gómez \& Gómez, 2011, p.187).

Los criterios expuestos anteriormente evidencian que dentro de los Programas de Estudio Ética, Estética y Ciudadanía para la Educación Cívica, se encuentran varias corrientes epistemológicas que tratan de brindar un panorama de trabajo y una justificación filosófica para este documento y la implementación de éste.. El análisis arroja que para la creación de estos programas se cuenta con poca información y formación en sus creadores, se denota la falta de especialistas en la elaboración y una capacitación deficiente para los educadores que deban implementar el programa.

En Costa Rica, el Proyecto Alfabetización Crítica en la Cultura Escolar: Cultura Política en la Secundaria Costarricense, ha realizado investigaciones que demuestran que los docentes, en muchos de los casos, no tienen la preparación ni la capacitación adecuada para lograr la "mejor" implementación de los programas de estudio, "se ha observado cómo se propicia la dominación de los docentes a través de la legislación emitida por el Ministerio de Educación Pública (MEP)" (Gómez \& Gómez, 2011, p.186), esto indica que los planes de estudio no sólo están 
creados para someter a los estudiantes, sino también para ser un medio de dominación a los encargados de "brindar" el conocimiento.

Es importante destacar que los objetivos son difusos y hacen imposible la vinculación de los estudiantes con lo que se pretende lograr. Por tanto, por un lado se dice que se pretende formar un tipo de estudiante con ciertas características, pero epistemológicamente se vuelve inviable lograrlo por mezclar teorías y por llevar a la práctica corrientes que no son eclécticas, entendiendo que de cierto modo se cae en una retórica que no va más allá del discurso, por ejemplo,

Como teoría epistemológica, el constructivismo es el elemento que permite definir con mayor claridad las formas de estimulación del aprendizaje del estudiantado, por lo que se desarrolla con mayor amplitud en este momento, complementado con algunos elementos del enfoque socio - reconstructivista. Ambos enfoques se asumen desde una visión que permite reflejar los elementos del humanismo, el racionalismo y el propio constructivismo como visiones filosóficas que les sirven de fundamento. (MEP, 2009, p.17)

Es pertinente recordar que para que una corriente sea ecléctica es necesario que sus enfoques epistemológicos sean compatibles entre sí, lo cual no es cumplido por el programa, aunado a ello y como muestra de un discurso que presenta un cúmulo de teorías que no tienen la más mínima compatibilidad se presenta la cita anterior sobre el constructivismo, en la cual se ratifica que el programa carece de un sustento epistemológico coherente, ya que la teoría pedagógica constructivista de Lev Vygotsky esta fundamentada sobre la base del materialismo dialéctico, el cual es discordante con lo que plantean las teorías mencionadas anteriormente, pues, según esta teoría, el estudiante podría crear su aprendizaje mediante la interacción con las personas y el maestro sería un culturalizador y/o facilitador, contrario a lo que las demás teorías presentes postulan, en donde el docente se convierte en el único poseedor del conocimiento y la verdad absoluta.

Desde este panorama, se puede determinar que el programa Ética, Estética y Ciudadanía para la asignatura de Educación Cívica, se ubica en la teoría pedagógica llamada conductismo, la cual tiene sus bases epistemológicas en el pragmatismo y el idealismo, con eso se puede 
indicar que el conductismo es básicamente antidialéctico ya que dicho modelo, no muestra interés por la conciencia, ni por la transformación cualitativa del conocimiento de los estudiantes; en otras palabras, tiene como objeto el contenido y/o objeto y no el sujeto, por ello impide que los estudiantes puedan problematizar los contenidos brindados por el educador, menos aún poder lograr reflexiones críticas de acuerdo con sus experiencias previas.

El programa de Educación Cívica que se está implementando en los colegios de secundaria en Costa Rica carece de sentido epistemológico y crea un conflicto de perspectivas, desde sus creadores hasta a los docentes que lo deben aplicar todos los días en las aulas de secundaria.

La imposibilidad de hacer efectivos los discursos que se plantean desde el mismo programa demuestra que la carencia de un marco filosófico coherente, no sólo pone en duda la veracidad de que el programa esté bien realizado, también demuestra que detrás de los programas de estudio se esconden sombríos fines vinculados a los intereses de sectores específicos de la sociedad, quienes con la formulación de Proyectos de Ética, Estética y Ciudadanía, pretenden cumplir ciertos objetivos para lograr desarrollar sus políticas de Estado y para ello necesitan un ciudadano que les sea afín, que carezca de una capacidad de reflexionar críticamente; uno que no cuestione lo que su docente le enseña en las aulas, un ciudadano incapaz de emanciparse de las estructuras simbólicas de poder que desde la escuela le incitan a obedecer y creer, que entre en consonancia con lo planteado por el sistema y la globalización, que buscan moldear a todos para estandarizarlos y hacerlos más dóciles hacia lo que necesita y exige el mercado y el consumo.

A pesar de este panorama incierto, se debe ser consciente de que existen alternativas que pueden evitar que teorías tan poco pedagógicas lleguen a las aulas del sistema educativo en Costa Rica, pero estas solo se pueden lograr si se aceptan con compromiso y responsabilidad los retos queimplican para todos aquellos que quieran generar un verdadero cambio en la educación. Por ello se vuelve fundamental y prioritario que las facultades de educación y todas aquellas con carreras afines, vuelvan su mirada a esta situación y comiencen a incorporar dentro de las mallas curriculares de las carreras, cursos de filosofía de la educación y epistemología. Los educadores deben tener

muy claras las concepciones filosóficas presentes en los programas de estudio; pues a partir de ellas o las que se decida aplicar, se 
determinará qué tipo de conocimiento y qué resultados se quieren obtener, es decir, el cómo y el para qué, se creará tal conocimiento.

Al docente poseer una compresión amplia sobre los procesos de creación del conocimiento, podrá tener dominio de las corrientes epistemológicas, de esta manera podrá subsanar las contradicciones filosóficas que están presentes en los programas del MEP. En se sentido, se vuelve prioritario darle a la educación, de nuevo el sustento filosófico que en algún determinado momento del pasado tuvo, "recuperar la etapas abandonadas de la reflexión, someter a examen crítico la concepción positivista del conocimiento y de la ciencia, es absolutamente necesario para cualquier forma de praxis que trate de promover la emancipación humana" (Carmona, 2007, s.p.).

Se ha demostrado a lo largo del texto, que los programas de estudio no presentan coherencia en su discurso, ni son concordantes con las teorías filosóficas que dicen estarse implementando, y que de una u otra manera lo único que logran es atrofiar el proceso de lo deseado y lo logrado en el sistema educativo.

Por ello se vuelve una necesidad la construcción de nuevos programas de estudio, que reflejen coherencia entre sus fundamentos filosóficos. Aunado a ello, se debe entender que para que verdaderamente se incorporen las concepciones socioculturales de las diferentes etnias de Costa Rica, se deben crear programas que estén apegados al contexto y sean filosóficamente compatibles con la manera en que las poblaciones tienen para construir y apropiarse del conocimiento.

\section{Referencias bibliográficas}

Bunge, M. (1981). Epistemología. Barcelona: Ariel.

Carmona, M. (2007). La formación filosófica del docente en Venezuela a través del programa de filosofía para niños. Revista Episteme, 27 (1).

Gómez, J. (2012).Guía de trabajo para evaluar la Teoría Sociohistórica de Vygotsky. (Trabajo sin publicar).

Gómez, J. (2012). Pedagogía del Conocimiento. Recuperado de www.unapedagogiadelconocimiento.blogspot.com.

Gómez, J. \& Gómez, L. (2011). Elementos teóricos y prácticos de la PedagogíaCrítica: Más allá de la Educación, Metáfora, Escena y Experiencia. Revista Praxis, 66.

Ministerio de Educación Pública de Costa Rica. (2009). Proyecto de Ética, Estética y Ciudadanía, Programas de Estudio, Educación Cívica, Tercer Ciclo de Educación General Básica y Educación Diversificada. 